\title{
A cardiovascular collapse occurred in the beach chair position for shoulder arthroscopy under general anesthesia -A case report-
}

\author{
Jihyun So, Woo-Jong Shin, and Jae-Hang Shim \\ Department of Anesthesiology and Pain Medicine, College of Medicine, Hanyang University, Guri, Korea
}

The occurrence of severe hypotension and bradycardia, following placing to the beach chair position from supine during general anesthesia for repair of tendon injury of the rotator cuff of shoulder in a healthy 50 year-old man was described. The Bezold-Jarisch reflex, which is known to inhibit cardiovascular reflex and composed of three kinds of symptoms such as vasodilation, bradycardia and hypotension, has been reported mainly in peripheral nerve block, and may occur during orthostasis, hypovolemia, hemorrhage, supine inferior vena cava compression in pregnancy, interscalene block for shoulder surgery in the sitting position and so on. The bradycardia and hypotension can be more aggravated when causative elements overlaps each other. Anticholinergics and vasopressor were injected intravenously, and position of the patient was changed to the supine position immediately resulting in a normal vital signs dramatically. (Korean J Anesthesiol 2013; 64: 265-267)

Key Words: Bradycardia, General anesthesia, Position, Reflex.

Several factors have been reported to be the cause of cardiovascular collapse. Cardiovascular collapses in the orthostatic position, hypovolemia, spinal and neuroaxial anesthesia, shoulder joint surgery in the sitting position under interscalene block, and general anesthesia related to pain and anxiety during venipuncture arose from Bezold-Jarisch reflex (BJR) activation. When these factors take place together, additional effects are generated. Inhalation anesthetics reduce the venous return since they have a direct vasorelaxation effect [1]. In the beach chair position, decreased venous return becomes more severe from vasorelaxation and myocardial depression effects of inhalation anesthetics. Blood pressure is regulated to maintain hemodynamic stability by various mechanisms [2]. BJR is a cardioinhibitory reflex that maintains tonic oppression in the vasomotor center [3]. BJR is defined as the state where heart rate is reduced to less than $50 / \mathrm{min}, 30 /$ min over five minutes, or where the systolic arterial pressure is less than $90 \mathrm{mmHg}$ [3]. BJR is activated by various stimuli including hypovolemia, redistribution of blood volume, mental stress, phobia, pain, parasympathetic system activation and

Received: February 22, 2012. Revised: 1st, April 18, 2012; 2nd, May 30, 2012. Accepted: June 5, 2012.

Corresponding author: Woo-Jong Shin, M.D., Ph.D., Department of Anesthesiology and Pain Medicine, Hanyang University Guri Hospital, 249-1, Gyomoon-dong, Guri 471-701, Korea. Tel: 82-31-560-2390, Fax: 82-31-563-1731, E-mail: swj0208@hanyang.ac.kr

(c) This is an open-access article distributed under the terms of the Creative Commons Attribution Non-Commercial License (http:// creativecommons.org/licenses/by-nc/3.0/), which permits unrestricted non-commercial use, distribution, and reproduction in any medium, provided the original work is properly cited. 
sympathetic system repression $[3,4]$.

After getting written consent from patients family member, we report the case of a healthy 50 -year-old male patient in the beach chair position to undergo shoulder joint surgery under general anesthesia using sevoflurane and suddenly showed severe bradycardia and hypotension.

\section{Case Report}

The patient was male, aged 50, $169 \mathrm{~cm}$ and $72 \mathrm{~kg}$, and visited the hospital to undergo shoulder joint surgery with a damaged in the right rotator cuff. Although he had diabetes that was diagnosed ten years before, he did not undergo treatment for it; however, blood sugar level had been controlled. Preoperative endocrinologic evaluation revealed no abnormal findings. The patient sometimes complained of palpitations and underwent tests for that in another hospital, but no anomaly was found and he was being followed-up. Nonspecific ST-T wave change was found in the preoperative electrocardiograph. No specific findings were found in the chest radiograph or echocardiogram, and laboratory findings were normal. Premedication was not administered. Vital signs just before induction and after arriving in the operation room were as follows: blood pressure of 160/90 $\mathrm{mmHg}$, heart rate of $70 / \mathrm{min}$, and oxygen saturation of $97 \%$, and electrocardiography showed normal sinus rhythm. Anesthesia was induced with pentothal sodium $300 \mathrm{mg}$, fentanyl $100 \mu \mathrm{g}$, and rocuronium bromide $50 \mathrm{mg}$, and the endotracheal tube (inner diameter $8.0 \mathrm{~mm}$ ) was inserted orally. For maintenance of anesthesia, $50 \% \mathrm{~N}_{2} \mathrm{O}-\mathrm{O}_{2}$, sevoflurane 3 vol\%, and remifentanil $0.05 \mu \mathrm{g} / \mathrm{kg} / \mathrm{min}$ were used. After vital signs were stabilized, concentration of sevoflurane was adjusted to 1.5 vol\% and atropine sulfate $0.5 \mathrm{mg}$ was intravenously injected just before changing the patient's position from supine to a sitting position (beach chair position $70-80^{\circ}$ ). One or two minutes after sitting position, heart rate was suddenly reduced from 80 / $\mathrm{min}$ to $30 / \mathrm{min}$ or less, and blood pressure was $70 / 40 \mathrm{mmHg}$. But no electrocardiograhic changes were noted. Intravenous injection of atropine sulfate $0.5 \mathrm{mg}$ was immediately given, but heart rate and blood pressure did not change. After stopping the administration of remifentanil and sevoflurane, $1: 10,000$ epinephrine $1 \mathrm{ml}(10 \mu \mathrm{g})$ was intravenously injected, and the patient was moved to the supine position. Heart rate soon recovered to $90 / \mathrm{min}$, and blood pressure returned to $120 / 60$ $\mathrm{mmHg}$. The results of the arterial blood gas analysis at that time were within normal range. Through the discussion with surgeons, we decided to postpone the operation. Detailed tests were performed a 24-hour electrocardiography and echocardiography, and the patient was suspected of having vasovagal syncope suspect. The head-up tilt testing and circulatory assessment diagnosed BJR as the cause. A sufficient amount of fluid was supplied and a $\beta$-sympatholytic was orally administered as the preventive treatment. Following preoperative administration of anticholinergic and sedative, anesthesia was induced well with desflurane and remifentanil. Afterwards, the operation was performed without any specific findings.

\section{Discussion}

The hypotension accompanied by bradycardia in this case may appear similar to syncope (i.e., BJR activation). BJR is overlapped by vasovagal syncope, one type of neurocardiogenic syncope, which is caused by reduction of venous return to the heart due to the decrease of peripheral arterial pressure by the neurogenic mechanism [2]. BJR is a reflex action that causes three signs: vasorelaxation, bradycardia, and hypotension [3]. Lower extremity muscle contraction is necessary in maintaining venous return to the heart and blood pressure [3]. General anesthesia as well as epidural anesthesia inhibits such a "muscle pump" and the compensatory arterial and venous constriction. Syncope due to the phobia and pain during general induction has been reported sometimes [5,6]; however, it is relatively rare in general anesthesia. Venous congestion caused by the sitting position during shoulder joint surgery under general anesthesia brings about the decrease of intraventricular volume, and rapid preload decrease causes abnormal myocardial contraction by the hypersecretion of catecholamine [4,7]. Enhanced myocardial contraction under reduced preload activates mechanical receptors of the heart, abnormally activates parasympathetic system, and brings about repression of sympathetic system. As a result, bradycardia is caused because of reflex vasorelaxation and vagus nerve activation. Paradoxical hypotension and bradycardia can be caused by intracardiac BJR activation because of blood flow dysfunction and vasodilator due to venous blood stasis and the insufficiency of intracardiac blood that leads to vigorous cardiac contraction [2]. When a headup tilt test is performed, it takes approximately $12-24$ minutes until hemodynamic changes, including the decrease of mean arterial blood pressure and heart rate begin, but it may take about one hour if the position is tilted by approximately $60^{\circ}[4]$. In this case, severe bradycardia started immediately when the sitting position was taken about 20 minutes after induction. There are two types of vasovagal inhibition: vasodilatory (VD) and cardioinhibitory (CI) type [8]. The VD type consists of a drastic reduction of arterial blood pressure without bradycardia, whereas the CI type is characterized by bradycardia associated with hypotension. The reported frequency of bradycardia and hypotension in the sitting position during interscalene block has been $13-29 \%$ [4,9-11]. The severity of bradycardia and hypotension varies ranging from a mild state to cardiac arrest 
and severe complications such as temporary loss of vision, cerebral and spinal ischemia, and ophthalmoplegia [1].

Appropriate fluid and $\beta$-sympatholytic should be intravenously injected to maintain sufficient volume of blood before BJR takes place, and preventive anticholinergic administration should be performed to repress the vasovagal reflex $[12,13]$. Moreover, the venous return volume should be immediately supplemented by altering position from the sitting to the supine position. Total administered fluid during the operation were about $150 \mathrm{ml}$ in this case. The cephalic region should be lowered and the lower extremity should be lifted up. The reduced ventricular volume caused by the orthostatic position and excessive myocardial contraction induce BJR by stimulating the intracardiac mechanical receptors. Even though atropine sulfate has been used as the only drug to treat the asystole that takes place under general anesthesia, it was not effective in our case [3]. Sympathomimetic drugs, such as ephedrine and various selective $\alpha$-agonists, repress venous and arterial vasorelaxation, and the rapid injection of epinephrine has been emphasized as an important factor of successive treatment [3].

In various studies on the treatment of vasovagal syncope, it was reported that atropine sulfate had efficacy in the CI type but not the VD type, and that studies had not been conducted yet with a sufficient number of patient to make a conclusion with respect to the effectiveness of vasovagal syncope treatment [8].

Currently, treatment methods for relapsing vasovagal syncope are divided into long-term, oral drug and pacemaking [8]. These treatment methods serve different purposes: drug treatment is effective in inhibiting initiation of the vasovagal reflex, whereas pacemaking can eliminate the reflex action immediately after symptom appears.

Based on this case, syncope should be examined in a patient who is to undergo the shoulder joint surgery in the sitting position under general anesthesia. If suspected, specific tests such as head-up tilt testing may be necessary. Additionally, in the case of suspected syncope by intraoperative positional change or other causes, the patient should be quickly moved to the supine position, and the selection of appropriate drugs should be taken into consideration.

\section{References}

1. Pohl A, Cullen DJ. Cerebral ischemia during shoulder surgery in the upright position: a case series. J Clin Anesth 2005; 17: 463-9.

2. Campagna JA, Carter C. Clinical relevance of the Bezold-Jarisch reflex. Anesthesiology 2003; 98: 1250-60.

3. Kinsella SM, Tuckey JP. Perioperative bradycardia and asystole: relationship to vasovagal syncope and the Bezold-Jarisch reflex. Br J Anaesth 2001; 86: 859-68.

4. D'Alessio JG, Weller RS, Rosenblum M. Activation of the BezoldJarisch reflex in the sitting position for shoulder arthroscopy using interscalene block. Anesth Analg 1995; 80: 1158-62.

5. Hampl KF, Schneider MC. Vasovagal asystole before induction of general anaesthesia. Eur J Anaesthesiol 1994; 11: 131-3.

6. Hart PS, Yanny W. Needle phobia and malignant vasovagal syndrome. Anaesthesia 1998; 53: 1002-4.

7. Ammirati F, Colivicchi F, Di Battista G, Garelli FF, Santini M. Electroencephalographic correlates of vasovagal syncope induced by head-up tilt testing. Stroke 1998; 29: 2347-51.

8. Santini M, Ammirati F, Colivicchi F, Gentilucci G, Guido V. The effect of atropine in vasovagal syncope induced by head-up tilt testing. Eur Heart J 1999; 20: 1745-51.

9. Kahn RL, Hargett MJ. Beta-adrenergic blockers and vasovagal episodes during shoulder surgery in the sitting position under interscalene block. Anesth Analg 1999; 88: 378-81.

10. Liguori GA, Kahn RL, Gordon J, Gordon MA, Urban MK. The use of metoprolol and glycopyrrolate to prevent hypotensive/bradycardic events during shoulder arthroscopy in the sitting position under interscalene block. Anesth Analg 1998; 87: 1320-5.

11. Sia S, Sarro F, Lepri A, Bartoli M. The effect of exogenous epinephrine on the incidence of hypotensive/bradycardic events during shoulder surgery in the sitting position during interscalene block. Anesth Analg 2003; 97: 583-8.

12. Batra AS, Balaji S. Usefulness of tilt testing in children with syncope: a survey of pediatric electrophysiologists. Indian Pacing Electrophysiol J 2008; 8: 242-6.

13. Lee HW. Hypotensive and bradycardic episodes in the sitting position during shoulder arthroscopy using interscalene block: can those be alerted? Korean J Anesthesiol 2010; 58: 1-3. 